\title{
Preparation, Administration, and Assessment of In vivo Tissue-Specific Cellular Uptake of Fluorescent Dye- Labeled Liposomes
}

\author{
Victoria Osinski ${ }^{1,2}$, Alexander L Klibanov ${ }^{1,3}$, Coleen A McNamara ${ }^{1,3}$ \\ ${ }^{1}$ Robert M. Berne Cardiovascular Research Center, University of Virginia ${ }^{2}$ Department of Pathology, University of Virginia ${ }^{3}$ Department of Medicine, \\ Division of Cardiovascular Medicine, University of Virginia
}

\section{Corresponding Author}

Victoria Osinski

vo3sc@virginia.edu

\section{Citation}

Osinski, V., Klibanov, A.L.,

McNamara, C.A. Preparation,

Administration, and Assessment

of In vivo Tissue-Specific Cellular

Uptake of Fluorescent Dye-Labeled

Liposomes. J. Vis. Exp. (161), e61585, doi:10.3791/61585 (2020).

\section{Date Published}

July 30,2020

DOI

$10.3791 / 61585$

URL

jove.com/video/61585

\section{Abstract}

There is a growing interest in using liposomes to deliver compounds in vivo particularly for targeted treatment approaches. Depending on the liposome formulation, liposomes may be preferentially taken up by different cell types in the body. This may influence the efficacy of the therapeutic particle as progression of different diseases is tissueand cell-type-specific. In this protocol, we present one method for synthesizing and fluorescently labeling liposomes using DSPC, cholesterol, and PEG-2000 DSPE and the lipid dye DiD as a fluorescent label. This protocol also presents an approach for delivering liposomes in vivo and assessing cell-specific uptake of liposomes using flow cytometry. This approach can be used to determine the types of cells that take up liposomes and quantify the distribution and proportion of liposome-uptake across cell types and tissues. While not mentioned in this protocol, additional assays such as immunofluorescence and single-cell fluorescence imaging on a cytometer will strengthen any findings or conclusions made as they permit assessment of intracellular staining. Protocols may also need to be adapted depending on the tissue(s) of interest.

\section{Introduction}

As interest in developing therapies utilizing nanoparticles drug delivery grows, the methods to prepare and assess particle distribution and uptake must continue to advance, expand, and be accessible to the research community ${ }^{1,2}$. This protocol was developed to assess the exact cell types that took up liposomes in vivo following a treatment with DiD-labeled liposomes loaded with tesaglitazar, a peroxisome proliferator-activated receptor (PPAR)- $\alpha / \mathrm{Y}$ agonist ${ }^{3,4}$. In these studies, we were able to assess which cell types were directly impacted by liposomal tesaglitazar treatment, the efficacy of targeting moieties, and generate hypotheses to explain the treatment outcomes we observed. Furthermore, established biological functions in a variety of cell types suggest that phagocytic cells such as macrophages, 
dendritic cells, and liver-specific Kupffer cells take up most of the liposomes ${ }^{5,6,7}$. Utilizing this protocol, we have demonstrated that non-classical phagocytes could also take up liposomes ${ }^{3,4}$.

This protocol presents an optimized method for solubilizing tesaglitazar, preparing liposomes by reversephase evaporation, and using calcium acetate as an attractant for remote drug loading. The methods presented are accessible to many labs and lack hard-to-acquire materials and steps requiring high temperatures. The protocol produces liposomes of sizes which are optimal for increased circulation in vivo ${ }^{8}$. Furthermore, as summarized by Su et al., to date, methods to evaluate in vivo liposome distribution and tissue uptake have been studied and tested in depth ${ }^{9}$. Positron emission tomography (PET), magnetic resonance imaging (MRI) and fluorescence molecular tomography (FMT) methods are applied to quantify tissue-specific biodistribution and uptake ${ }^{9}, 10,11$. While these methods have been optimized to maximize detection in vivo, they still lack the ability to quantify liposome uptake in vivo at cellular resolution. The protocol presented here aims to accomplish this need through the use of flow cytometry. Finally, for this protocol, cellular uptake was narrowed down to a few tissues including adipose tissue. There is a growing body of literature investigating the potential for use of nanoparticles to deliver therapies in the setting of obesity, dysmetabolism, and inflammation $12,13,14,15,16,17$. As such, we felt it important to share a protocol with effective methods for processing and analyzing adipose tissue-one of the tissues that plays an important role in these pathologies.

\section{Protocol}

All steps in this protocol are approved by and follow the guidelines of the Animal Care and Use Committee at the University of Virginia.

NOTE: There are some important controls to consider for later analysis steps, which are summarized in Table 1 and should be considered prior to liposome administration.

\section{Preparation of fluorescently labelled liposomes, loaded with calcium acetate and tesaglitazar}

1. Combine DSPC (1,2-distearoyl-sn-glycero-3phosphocholine), cholesterol, PEG-2000-DSPE, and DiD. For this, combime the DSPC, cholesterol, and PEG-2000 DSPE at a mass ratio of 2:1:1. Add DiD lipid dye at a concentration of $1 \mathrm{mg}$ of DiD per $1 \mathrm{~mL}$ of liposomes (molar ratio of 46:1 of DSPC:DiD).

NOTE: $\mathrm{DiD}$ is an accepted abbreviation for 1,1'dioctadecyl-3,3,3',3'tetramethylindocarbocyanine dye. As it has two octadecyl "fatty tails" of equal length to the DSPC utilized in this formulation, it should mostly incorporate into the lipid membrane. Lipid dyes like DiO, DiD and Dil are routinely used for liposome research ${ }^{8}$ and they are considered non-exchangeable ${ }^{18}$.

2. Use a $20 \mathrm{~mL}$ scintillation vial for the inverted phase emulsion and liposome preparation. In this vial, mix a 2:1 ether-chloroform solution of lipids with aqueous calcium acetate (Ca-acetate, $1 \mathrm{M}, \mathrm{pH}$ 7.4). The ratio between organic and aqueous phase should be $4: 1$, e.g., $4 \mathrm{~mL}$ of organic phase and $1 \mathrm{~mL}$ of aqueous phase.

3. Emulsify the ether-chloroform solution of lipids by sonication for $30 \mathrm{~s}$ at room temperature. Operate the 
sonicator at $20 \mathrm{KHz}$ and $50 \%$ power and use a $1 / 2$ in. probe.

NOTE: Keep the tip of the sonicator probe closer to the bottom of the vial to avoid foaming. Do not touch the glass with the probe tip during sonication, it may break. Additionally, chloroform needs to be added to the ether as a co-solvent: in the presence of cholesterol, an etheronly emulsion separates rapidly, making this step of the procedure impossible.

4. Immediately place the vial with homogenized water-in-oil emulsion on a rotary evaporator with a special adapter, manometer gauge, and a pressure regulator valve. The evaporator should be connected to a vacuum line to remove the organic solvents. Set the rotation rate at $100 \mathrm{rpm}$ and the vacuum at $0.5 \mathrm{~atm}$, and release if emulsion foaming looks excessive. After a gel forms and disappears, increase the vacuum to $0.9 \mathrm{~atm}$.

NOTE: During volatile organic phase removal, the vacuum level should be adjusted gradually, to avoid rapid foaming, as it may lead to content loss from the vial into the body of the rotary evaporator. Eventually, when the ether and chloroform partially evaporate and the volume ratio between aqueous and organic solvent phase is close to 1:1, a gel will form. Evaporation should continue until the gel disappears and remaining aqueous media is completely liquid again. Additional mixing may help accelerate organic solvent removal. This can be achieved by placing a polytetrafluoroethylene stir bar into the evaporation flask, to enhance convection of the viscous gel during rotary evaporation.

5. Filter the resultant liposomes using track-etched polycarbonate membranes to achieve homogeneous size distribution.
1. Perform filtration by passing the liposome aqueous dispersion back-and-forth multiple times through a $200 \mathrm{~nm}$-pore polycarbonate filter in a liposome extruder equipped with two gas-tight syringes.

NOTE: Smaller syringes are preferred (e.g., $0.5 \mathrm{~mL}$ ) as they assure generation of sufficient pressure for filtration. With a high cholesterol content in the liposome membrane, a high temperature is not necessary, and the procedure can be performed at room temperature. An odd number of filtrations (e.g., 21 ) is performed, so that the resulting material ends up on the opposite side of the filter from the start and if pre-sterilized, the sterile sample of filtered size adjusted-liposomes can be collected. The size of the resulting liposomes is typically close to the selected filter pore size. Two filters can be stacked (instead of one) to perform fine adjustment to lower particle size.

2. Verify size distribution using dynamic laser light scattering $(\mathrm{DLS})^{3,4}$.

1. Add 1 to $3 \mathrm{~mL}$ of saline to a $1 \mathrm{~cm}$ cuvette with four transparent sides. To that, add 10$20 \mu \mathrm{L}$ of liposomes and mix carefully. Place the sample into the apparatus and select the following parameters to measure: solvent viscosity, refractive index, refractive index of lipids. Click the Start button. The measurements will last several minutes and consist of 100 or more runs.

6. Remove external Ca-acetate using a desalting spincolumn. To half of the batch, add aqueous tesaglitazar in 10 mM HEPES buffer ( $\mathrm{pH}$ 7.4) and incubate with mixing at $37^{\circ} \mathrm{C}$ for $1 \mathrm{~h}$. Use the second half of the batch as a drug-free control liposome formulation. 
NOTE: Pre-equilibrate the 2-mL desalting spin-column with $10 \mathrm{mM}$ HEPES buffer, $\mathrm{pH}$ 7.4, prior to use. To do this, place $1 \mathrm{~mL}$ of HEPES buffer into the column and spin in a centrifuge at $1000 \times g$ for $2 \mathrm{~min}$. Remove the passthrough buffer and repeat this four times.

7. Remove unentrapped tesaglitazar from liposomes using a $2 \mathrm{~mL}$ spin-column, and determine the concentration of entrapped drug spectrophotometrically.

8. Add no more than $0.5 \mathrm{~mL}$ of liposome sample to the dry column gel bed and wait until all the sample enters the gel. Centrifuge at exactly the same conditions as earlier (1000 $x \mathrm{~g}, 2 \mathrm{~min}$ ) and collect the liposome sample in the passthrough purified from small molecular mass compounds.

9. Quantify final particle features: particle size and concentration using DLS and zeta potential with a combined DLS-electrophorectic light scattering (ELS) system ${ }^{3,4}$ in $10 \mathrm{mM} \mathrm{HEPES}$ buffer $\mathrm{pH} 7.4$ and at $25^{\circ} \mathrm{C}$.

1. Similar to step 1.5.2, dilute liposome dispersion in the measurement buffer (e.g., $10 \mu \mathrm{L}$ liposomes per $1 \mathrm{~mL}$ of buffer solution) into a U-shaped cuvette using a disposable Luer syringe, or a pipette with a cut tip. Make sure there are no bubbles in the "U" so that there is uninterrupted solution for electrical current flow.

2. Place the cuvette into the unit (please pay attention to the front and back of the cuvette, so that electrodes are properly connected to the unit). Close the instrument door; after this, the measurement takes place (with multiple repeats), under control of the guidance software.

\section{Prepare liposomes for in vivo administration}

1. In a biosafety cabinet, dilute liposomes in sterile saline to the appropriate concentration in a final volume of $50 \mu \mathrm{L}$ for in vivo administration.

NOTE: In previous studies, our liposome preparation contained $2 \mathrm{mg} / \mathrm{mL}$ of tesaglitazar, which equals about 4.89 $\mu \mathrm{mol}$ of tesaglitazar $/ \mathrm{mL}$, and we administered liposomes at a dose of $1 \mu \mathrm{mol}$ drug/kg. For a $40 \mathrm{~g}$ mouse, we would bring $8.2 \mu \mathrm{L}$ of liposomes up to a final volume of $50 \mu \mathrm{L}$ in saline. Using DLS/ELS, the number of liposomes per unit volume should also be quantified for preparations of drug- and vehicle-loaded liposomes to ensure that an equal number of vehicle liposomes are administered per gram of mouse weight compared to the drug-loaded liposomes.

2. Load the liposome solution into a $27 \mathrm{G}$ needle in the biosafety cabinet. Keep this at room temperature to avoid injecting cold solution into the mouse.

\section{Administer liposomes via retro-orbital intravenous injection}

NOTE: It is also appropriate to conduct the intravenous injection by other methods, such as tail vein injections if it is preferred. While not covered in this protocol published protocols explaining this method ${ }^{19}$ are available.

1. Set up the workspace for delivering liposomes.

1. Clean the workbench with $70 \%$ ethanol. Make sure to select a space that permits the use of an isoflurane anesthesia system.

2. Turn on a warming pad and place a clean pad or towel over it to keep the mouse on a clean surface. Allow 
enough time for the pad to warm up before beginning work with mice.

3. Set up the anesthesia system so that the chamber is nearby and the nose cone is on the warming pad.

1. Make sure all other aspects of the system are ready (for example, isoflurane level is high enough in the vaporizer, the charcoal filter has been weighed, tubing is connected correctly).

4. Gather the other materials needed for this section of the protocol: ophthalmic lubricant gel, a local anesthetic for post-administration treatment, sterile gauze pads.

2. Sedate the mouse using isoflurane in the induction chamber. Once it is unresponsive to a gentle foot tap, quickly transfer the mouse to the workspace while maintaining sedation through a nose cone.

3. Shift the mouse to one side for liposome administration. Because the mouse will not blink while anesthetized, apply a small amount of ophthalmic lubricant to keep the eye moisturized during the remainder of the procedure.

4. Gently press down on the skin above and below the exposed eye. The eye should lift above the plane of the face.

5. Carefully insert the tip of the needle at the front corner of the eye, making sure the needle is below the eye and not touching it. Once the needle is inserted below the eye, slowly inject the liposomes into the retro-orbital space.

1. If the needle is not inserted far enough in, the solution may emerge up around the eye. Stop injecting immediately if this is seen and re-position the needle.

6. Apply a local anesthetic, such as proparacaine, to the eye to prevent post-procedure pain and discomfort.
7. Keep the mouse on a warming pad and monitor until it awakens to ensure it is well and maintains proper body temperature.

8. Return the mouse to its cage and its normal housing environment until the time point of interest arrives.

NOTE: This should be done in line with local IACUC guidelines.

\section{Prepare materials for the tissue harvest, tissue processing, and flow cytometry staining}

1. Prepare solutions for the harvest, processing, and staining (sections 5-7): phosphate-buffered saline (PBS)Heparin, HEPES Buffer, $2 \mathrm{mg} / \mathrm{mL}$ Collagenase type I, AKC lysis buffer, FACS buffer, PBS, Fixation buffer (Table 2). Keep all solutions except the fixation buffer at $4{ }^{\circ} \mathrm{C}$ or on ice during the procedure.

2. Prepare tubes with buffers and other materials for harvesting and processing tissues.

1. For blood from each mouse, add $10 \mu \mathrm{L}$ of $0.5 \mathrm{M}$ EDTA to a 1.5 or $1.7 \mathrm{~mL}$ microcentrifuge tube for collecting the blood. The EDTA will prevent the blood from clotting. A $1 \mathrm{~mL}$ syringe with a $25 \mathrm{G}$ needle and a $15 \mathrm{~mL}$ conical tube are also needed.

2. For the spleen, gather one 1.5 or $1.7 \mathrm{~mL}$ microcentrifuge tube with $1 \mathrm{~mL}$ of HEPES buffer, a 1 $\mathrm{mL}$ syringe, two $50 \mathrm{~mL}$ conical tubes, and two $70 \mu \mathrm{m}$ filters per spleen.

3. For each adipose tissue depot, gather a $20 \mathrm{~mL}$ polyethylene vial with $1.5 \mathrm{~mL}$ of HEPES buffer for mincing the tissue, a $50 \mathrm{~mL}$ conical tube, and a $70 \mu \mathrm{m}$ filter per adipose tissue type per mouse.

3. Prepare the workspace for the harvest. 
1. Clean off the bench space with $70 \%$ ethanol. Prepare a rubber tray for pinning the mouse during harvest by cleaning it off with $70 \%$ ethanol and covering it with an absorbent pad or paper towels. Make sure at least 5 pins are available to work with.

2. Fill a $10 \mathrm{~mL}$ syringe with PBS-heparin and fasten on a $25 \mathrm{G}$ needle for perfusion.

3. Gather tools and materials to use during the harvest. Forceps (two pairs), scissors, paper towels, lint-free wipes, the microcentrifuge tube(s) with EDTA, the microcentrifuge tube(s) with HEPES buffer, and the polyethylene vial(s) with HEPES buffer are needed.

\section{Harvest the tissues}

1. Euthanize the mouse by $\mathrm{CO}_{2}$ asphyxiation. Do not conduct a cervical dislocation as this may prevent effective blood collection and tissue perfusion at later steps.

2. At a cleaned bench area with enough working space and lighting to see the mouse well, set up a rubber dissection tray, a bucket of ice for storing samples, and a spray bottle with $70 \%$ ethanol. Spray down the mouse with $70 \%$ ethanol to reduce contamination and control hair spread. Place the mouse on its back on the rubber tray and pin down its paws spread out away from its body.

3. To prepare for collecting blood, carefully make an incision in the skin at the edge of caudal end of the mouse's ribcage. Cut a small, straight line up towards the mouse's head (about $1 \mathrm{~cm}$ ) until the pectoralis muscles are exposed.

1. At the initial incision site, make two small cuts perpendicular to the line towards the head. Then, carefully cut away the pectoralis muscle on one side of the rib cage in the exposed area. This allows better access and visualization for where the needle should be inserted.

2. To collect blood, insert the needle between the third and fourth ribs on the side where the muscle was removed. Since the mouse's heart is found at the center of the chest cavity, keep the needle as close to the center line of the rib cage as possible. Once inserted, gently pull up on the syringe to begin collecting blood.

3. Once collected, transfer the blood to the prepared microcentrifuge tube with EDTA and store on ice.

NOTE: If about $100 \mu \mathrm{L}$ of volume is pulled up and no blood enters the syringe, try rotating the syringe to the right or left in case the needle opening is pressed up against the wall of the heart. If this does not help, slowly move the needle further into the chest cavity or begin removing. If blood begins to collect in the syringe at this point, continue to pull back on the syringe slowly. Consider rotating the syringe and needle for successful extraction. Finally, if no blood is collected, remove the needle as it may have missed the heart. Try reinserting the needle and repeating the aforementioned process again.

4. Next, to perfuse the mouse, open up the chest cavity to access the heart.

1. To do this, cut the skin along the end of the rib cage down to the mouse's side on each side. Then, use the forceps to hold up the sternum away from the working surface. Make a small, shallow incision just below the end of the sternum to cut through the peritoneal cavity. Cut along the peritoneal membrane along the end of the rib cage on each of the mouse's sides. This should 
expose the liver and gallbladder. Be careful not to cut into either of these tissues.

2. Next, make a small, shallow cut into the diaphragm, cranial to the liver. Then, cut the diaphragm along the edge of the rib cage to open up the chest cavity. Be sure to avoid cutting any of the organs within the chest cavity.

3. Make two cuts along the rib cage towards the head about 2-3 $\mathrm{mm}$ from the center line of the mouse and about $0.75 \mathrm{~cm}$ long.

NOTE: If cut up too high, the arteries residing at the top of the rib cage will be cut. This will interfere with efficacy of perfusion.

4. Lift back the center piece of the rib cage to expose the chest cavity. Move any fat or tissue away to access the heart.

5. Make a small cut into the right atrium of the mouse's heart to create an opening through which to push out the blood.

6. Using a $10 \mathrm{~mL}$ syringe of PBS-heparin, insert the needle into the left ventricle of the mouse's heart.

7. Gently begin pushing PBS into the heart as slowly as possible.

NOTE: Blood should be observed emerging from the right atria and filling the chest cavity. Be sure to keep the heart in its physiological location to avoid inhibiting the flow of PBS-heparin from the heart through the aorta.

8. Once all the $10 \mathrm{~mL}$ of PBS-heparin have been perfused through the mouse, discard the syringe and needle and remove excess blood and PBS-Heparin from the chest cavity using paper towels or lint-free wipes.
5. Next, to begin extracting tissues, cut down the skin and peritoneal membrane towards the mouse's tail to open up the peritoneal cavity.

6. First, extract the inguinal adipose tissue pad from each side of the mouse.

NOTE: Read this process carefully: be sure to extract the inguinal lymph node from each depot to avoid skewing the adipose tissue cellular make-up in the results.

1. Using a second set of forceps, hold the peritoneal membrane with one set of forceps and the edge of the skin overlaid above the membrane on that side with the other forceps. Gently pull the skin away from the peritoneal membrane to separate these layers from one another. Look for the the inguinal adipose tissue depot along the skin. Pin down the outer edge of the skin to better access the adipose depot.

2. Prior to extraction, locate the inguinal lymph node in the center of the adipose depot and remove it using forceps and scissors as needed.

NOTE: If possible, locate the three larger arteries that run from the outer edges of the depot towards the center. The lymph node is located around where these arteries meet.

3. After the lymph node is removed, carefully hold the end of the adipose depot nearest to the pinned point with the forceps and begin making small cuts at the connective membrane between the adipose tissue and the skin. Lift the adipose tissue away from the skin while making cuts to make better access the membrane and ensure the entire depot is extracted.

4. Place the adipose depot in a prepared polyethylene vial with HEPES buffer on ice to keep the tissue viable during the remainder of the harvest. 
5. Repeat this process on the other side of the mouse to extract both depots. Depots can either be digested and processed together or separately. If each depot is to be processed separately, more tubes must be prepared.

7. Next, extract the epididymal adipose depots from the caudal end of the peritoneal cavity. Using forceps, gently pull the first epididymal adipose depot way from the dorsal end of the mouse and locate the epididymis and vas deferens attached to this depot.

NOTE: There are two epididymal adipose depots: one attached to each epididymis and vas deferens.

1. Carefully cut between the adipose depot and the epididymis and vas deferens to separate the adipose from these other tissues. Place the adipose depot in a polyethylene vial with HEPES buffer on ice to keep the tissue viable during the remainder of the harvest.

8. Finally, extract the spleen, which is found to the left of the stomach near the diaphragm. Using forceps, gently pull the stomach towards the center of the peritoneal cavity to expose the spleen.

1. Gently hold one end of the spleen and pull it slightly away from the stomach. Cut the membrane between the spleen and its adjacent tissue until the organ is detached. Place the spleen in the prepared microcentrifuge tube with HEPES buffer and store on ice.

9. Prior to processing tissues or harvesting tissues from the next mouse, discard the carcass and any soiled paper towels or pads. Wipe off tools as well.

NOTE: If there are multiple mice, repeat these harvest steps for each mouse prior to moving on to the next processing step. If a control mouse/mice is included, consider harvesting these prior to liposome-treated mice to avoid any contamination.

\section{Process tissues}

NOTE: Since the adipose tissue has a long digestion incubation, it is recommended to start with that process first and work on processing the blood and spleen during the digestion period.

1. First, mince and digest the adipose tissues. Using one or two pairs of scissors, mince the adipose tissue in each polyethylene vial until the tissue is in small pieces of less than $0.5 \mathrm{~mm}$ in size. This allows for more efficient digestion.

1. Once tissues in all vials are minced, add $1.5 \mathrm{~mL}$ of $2 \mathrm{mg} / \mathrm{mL}$ Collagenase buffer to each vial. Place the vials in a shaking incubator set to $37^{\circ} \mathrm{C}$ and $150 \mathrm{rpm}$. Incubate for 30 to $45 \mathrm{~min}$.

NOTE: If the adipose tissues are particularly large, consider adding another $0.5 \mathrm{~mL}$ to $1.5 \mathrm{~mL}$ of HEPES buffer and an equal volume of Collagenase buffer to the vial(s) to ensure tissues are fully submerged and enough enzyme is present. The final concentration of Collagenase Type I at digestion should be $1 \mathrm{mg} / \mathrm{mL}$ regardless of the final solution volume. Furthermore, if a shaking incubator is not available, samples can be placed in a water bath heated to $37^{\circ} \mathrm{C}$. Gently shake the samples every $5 \mathrm{~min}$ to $\operatorname{mix}$ and resuspend the digestion.

2. Check the samples at $30 \mathrm{~min}$. Use a $1 \mathrm{~mL}$ pipet to pipet the sample up and down. If the tissue pieces are still too large for easy pipetting, return the samples to the incubator for an additional $15 \mathrm{~min}$. 
3. Once the samples are fully digested, continue to pipet the sample up and down another 10 times to ensure a single-cell suspension has been created.

NOTE: (Optional) Check the samples at $30 \mathrm{~min}$. Use a $1 \mathrm{~mL}$ pipette to pipet the sample up and down. If the tissue pieces are still too large for easy pipetting, return the samples to the incubator for an additional $15 \mathrm{~min}$.

4. Pipet the cell suspension through a $70 \mu \mathrm{m}$ filter into a $50 \mathrm{~mL}$ conical tube. Add $5 \mathrm{~mL}$ of FACS buffer to the empty digestion vial to wash the vial out. Transfer this wash buffer through the filter to add to the cell suspension.

5. Store samples on ice while others are being processed. Once all samples are filtered, spin them down at $400 \times g, 4{ }^{\circ} \mathrm{C}$ for $5 \mathrm{~min}$.

6. Remove the adipocyte supernatant by aspiration and then carefully remove the infranatant between the adipocyte supernatant and pellet by aspiration to leave the stromal-vascular fraction (SVF) pellet.

7. Resuspend this pellet in $1 \mathrm{~mL}$ of FACS buffer and transfer to a clean 1.5 or $1.7 \mathrm{~mL}$ microcentrifuge tube. Aliquot cells now if desired or needed. Keep on ice until all samples are ready for flow cytometry staining. NOTE: If the adipose depots digested were large, consider only using $50 \%$ or $25 \%$ of the sample for flow cytometric staining and analysis. Additionally, if any fluorescence-minus-one (FMO) controls or additional controls for flow cytometry analysis (Table 1) are needed, be sure to aliquot extra sample into a separate tube for processing. FMOs are used to distinguish between negative and positive signal for an individual fluorophore-conjugated antibody within the otherwise-complete panel utilized in the experiment.

2. Second, process the blood.

1. Transfer $50 \mu \mathrm{L}$ of blood to a $15-\mathrm{mL}$ conical tube.

2. Add $1 \mathrm{~mL}$ of AKC lysis buffer to each tube and pipet up and down to reach a single-cell suspension. Add an additional $4 \mathrm{~mL}$ of $\mathrm{AKC}$ lysis buffer to each tube and incubate for $5-10 \mathrm{~min}$. If a shaker or rotator is available, seal the tube caps tightly and place the tubes on one of these to enhance mixing.

3. Add $5 \mathrm{~mL}$ of FACS buffer to quench the lysis process and spin the samples at $400 \times \mathrm{g}, 4{ }^{\circ} \mathrm{C}$ for $5 \mathrm{~min}$. Remove the supernatant and check the pellet. If it is still quite red, repeat the lysis process. Otherwise, resuspend the pellets in $1 \mathrm{~mL}$ of FACS buffer and transfer to a clean 1.5 or $1.7 \mathrm{~mL}$ microcentrifuge tube. Keep on ice until all samples are ready for flow cytometry staining.

3. Finally, process the spleen. Transfer the spleen onto a $70 \mu \mathrm{m}$ filter over a $50 \mathrm{~mL}$ conical tube. Wash the tissue with $1 \mathrm{~mL}$ of FACS buffer and then mash the spleen through the filter using the plunger end of a $1 \mathrm{~mL}$ syringe. Throughout the mashing process, wash the cells into the $50 \mathrm{~mL}$ conical tube using more FACS buffer. The final volume in the conical tube should be $10 \mathrm{~mL}$.

1. Spin the cells at $300 \times g$ at $4{ }^{\circ} \mathrm{C}$ for $5 \mathrm{~min}$. Remove the supernatant and resuspend in $1 \mathrm{~mL}$ of AKC lysis buffer. Add an additional $4 \mathrm{~mL}$ of AKC lysis buffer and incubate for $5 \mathrm{~min}$. Add $5 \mathrm{~mL}$ of FACS buffer to quench the lysis process and spin the samples at $300 \times \mathrm{g}$ at $4{ }^{\circ} \mathrm{C}$ for $5 \mathrm{~min}$.

2. Remove the supernatant and resuspend the pellet in $1 \mathrm{~mL}$ of FACS buffer. Transfer the suspension through 
a second, clean $70 \mu \mathrm{m}$ filter into a $50 \mathrm{~mL}$ conical tube.

Add $4 \mathrm{~mL}$ of FACS buffer to wash out the original tube and transfer the buffer through the filter for a final volume of $5 \mathrm{~mL}$.

3. Transfer $50 \mu \mathrm{L}$ of the cell suspension to a clean 1.5 or $1.7 \mathrm{~mL}$ microcentrifuge tube and keep on ice until all samples are ready for flow cytometry staining. Additional aliquots can be transferred to tubes if more are desired or required.

NOTE: Splenocytes are excellent cells to use for a Live/Dead single stain. Consider transferring an additional aliquot for this control.

\section{Stain cells from tissues for flow cytometry}

1. Spin down aliquoted samples at $400 \times g, 4{ }^{\circ} \mathrm{C}$ for $5 \mathrm{~min}$.

2. Remove supernatant and resuspend samples in $50 \mu \mathrm{L}$ of Fc Block (diluted) (Table 2). Incubate on ice for $5 \mathrm{~min}$.

3. Add $50 \mu \mathrm{L}$ of $2 \mathrm{x}$ antibody mix (Table 3 ) to each sample. Incubate on ice in the dark for $20 \mathrm{~min}$.

NOTE: Any single stains should NOT be stained with this antibody mix. Additionally, if FMOs are to be used, FMO antibody mixes must be prepared separately.

4. Wash samples with $1 \mathrm{~mL}$ of PBS and spin at $400 \times \mathrm{g}$, $4{ }^{\circ} \mathrm{C}$ for $5 \mathrm{~min}$. Remove the supernatant and resuspend samples in $200 \mu \mathrm{L}$ of viability stain (Table 3 ). Incubate on ice in the dark for $20 \mathrm{~min}$.

NOTE: Do not forget to stain cells that were set aside for a Live/Dead single stain during this step.

5. Wash samples with $1 \mathrm{~mL}$ of FACS buffer and spin at 400 $\mathrm{x} g, 4^{\circ} \mathrm{C}$ for $5 \mathrm{~min}$. Remove supernatant and resuspend samples (except Live/Dead single stain) in $50 \mu \mathrm{L}$ of fixation medium (Reagent $A$ ) to fix samples. Incubate at room temperature in the dark for $15 \mathrm{~min}$.
1. Resuspend the Live/Dead single stain in $100 \mu \mathrm{L}$ of $2 \%$ PFA. Incubate at room temperature in the dark for 5 $\min$.

2. Wash sample with $1 \mathrm{~mL}$ of FACS buffer and spin at $800 \times \mathrm{g}, 4{ }^{\circ} \mathrm{C}$ for $5 \mathrm{~min}$. Remove supernatant and resuspend samples in 250 to $500 \mu \mathrm{L}$ of FACS buffer. Store at $4{ }^{\circ} \mathrm{C}$ until samples can be run on the flow cytometer.

6. Wash samples with $1 \mathrm{~mL}$ of FACS buffer and spin at $800 \mathrm{x}$ $\mathrm{g}, 4^{\circ} \mathrm{C}$ for $5 \mathrm{~min}$. Remove the supernatant and resuspend samples in $50 \mu \mathrm{L}$ of permeabilization medium (Reagent B) plus antibody/ies to intracellular proteins. Incubate at room temperature in the dark for $20 \mathrm{~min}$.

7. Wash the samples with $1 \mathrm{~mL}$ of FACS buffer and spin at $800 \times g$ at $4{ }^{\circ} \mathrm{C}$ for $5 \mathrm{~min}$. Remove the supernatant and resuspend the samples in $100 \mu \mathrm{L}$ of $2 \%$ paraformaldehyde (PFA). Incubate at room temperature in the dark for $5 \mathrm{~min}$.

8. Wash samples with $1 \mathrm{~mL}$ of FACS buffer and spin at $800 \mathrm{x}$ $\mathrm{g}, 4^{\circ} \mathrm{C}$ for $5 \mathrm{~min}$. Remove the supernatant and resuspend samples in 250 to $500 \mu \mathrm{L}$ of FACS buffer. Store at $4{ }^{\circ} \mathrm{C}$ until samples can be run on the flow cytometer.

\section{Representative Results}

\section{Liposome Production}

Results published here are similar to those in our previously published work ${ }^{3,4,20}$. Utilizing the protocol presented here, we expect to produce liposomes of approximately 150-160 $\mathrm{nm}$ in size. DLS reveals an average liposome diameter of $163.2 \mathrm{~nm}$ and a zeta potential of $-19.2 \mathrm{mV}$ (Figure 1A). Cryogenic electron microscopy (cryo-EM) imaging reveals circular liposomes (Figure 1B) and the DLS diagram reveals 
a relatively small standard deviation from the average diameter (Figure 1C).

\section{Positive liposome binding requires a PBS-treated control}

Prior studies from our group employing this protocol investigated what cell subsets in adipose SVF, spleen, and blood bound to liposomes following one week of in vivo administration $^{3,4}$. Using a PBS-treated mouse, peritoneal cavity and spleen cells were stained with the same antibody panel used on samples from liposome-treated mice. Tissues were harvested after one week of treatments (Figure 2A). The samples from the PBS-treated mouse served as a DiD FMO with which to create positive DiD gates (Figure 2B,C). A positive gate can be created using DiD-positive signal, but samples lacking DiD signal must also be used to verify that the positive gate does not include any DiD-negative samples.

\section{Titrations are needed to optimize fluorescence signals}

Prior to executing a full experiment, various conditions including the concentration of fluorescently conjugated antibodies used during cell staining and of lipid dye used during liposome preparation must be optimized. Flow cytometers have an upper limit of detection for fluorescence intensity, so too much dye incorporated in the liposomes will lead to unquantifiable levels of DiD signal in samples run through the cytometer. Furthermore, too much DiD in the liposomes may lead to high levels of non-specific dye transfer, which could skew cellular uptake results. Figure 3 reports results from an experiment in which concentrations of lipid dye were titrated to identify the concentration that would produce an optimal signal within the detection range of the flow cytometer that was used. This was conducted on the tissues of interest for the final experiment: Blood (Figure 3A), inguinal adipose SVF (Figure 3B), and epididymal adipose SVF (Figure 3C). The concentrations selected for testing were $10 \mathrm{mg}$ of $\mathrm{DiD}$ (High, red), $1 \mathrm{mg} \mathrm{DiD}$ (Middle, blue), or $0.1 \mathrm{mg}$ of DiD (Low, grey) per $1 \mathrm{~mL}$ of liposomes. The highest concentration used in the liposomes was too high and surpassed the quantifiable range of the cytometer in all three tissues (Figure $\mathbf{3 A}-\mathbf{C}$, red). The lowest concentration of DiD showed some signal (Figure 3A-C, grey), but a clear population beyond the PBS-treated cells (Figure 3A-C, black) was not observed. When quantified, the arithmetic mean of the DiD MFI for each tissue and concentration demonstrated a clear distinction between PBS controls and the middle concentration of DiD (Figure 3D). Thus, as indicated in the protocol, we selected the middle concentration (Figure 3, blue) to use in our liposome preparation.

The use of multi-antibody panel allows for identification of liposome uptake by different cell subsets

Using the panel outlined in Table 3, cells were stained with antibodies against markers for a macrophages, B cells, $T$ cells, dendritic cells, monocytes, and endothelial cells (Figure 4). Slightly different gating strategies are required for each tissue type, but most of the same cell types can be identified in each. Some exceptions include endothelial cells, which are not normally found in the blood, and monocytes, which are typically at higher frequency in the blood than other tissues. Once populations are identified, total size of each cell population and the frequency at which they are $\mathrm{DiD}^{+}$ can be quantified. Further calculations can be performed to characterize the $\mathrm{DiD}^{+}$population: what percent of $\mathrm{DiD}^{+}$cells are macrophages, endothelial cells, etc. Please note, these are example gating strategies, but not the only way to analyze the samples. Analysis will be dictated by the selected panel and flow cytometer(s) available. 


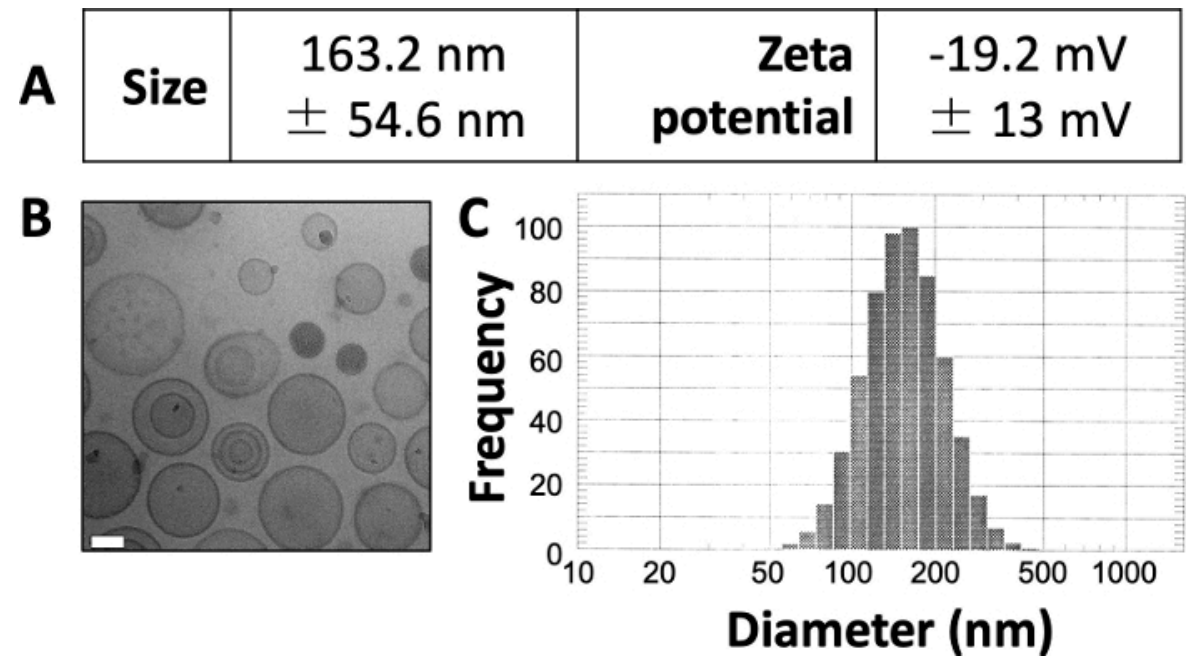

Figure 1: Example characteristics of prepared liposomes.

(A) The size and zeta potential were measured as described above and have been reported in table form. Each parameter is presented as the mean \pm the standard deviation. (B) Cryo-EM was used to image the prepared liposomes. The white scale bar is $50 \mathrm{~nm}$ in length. (C) DLS was used to generate a histogram of the diameter of liposomes in this prep. This figure is adapted from Osinski et al. ${ }^{3}$. Please click here to view a larger version of this figure.
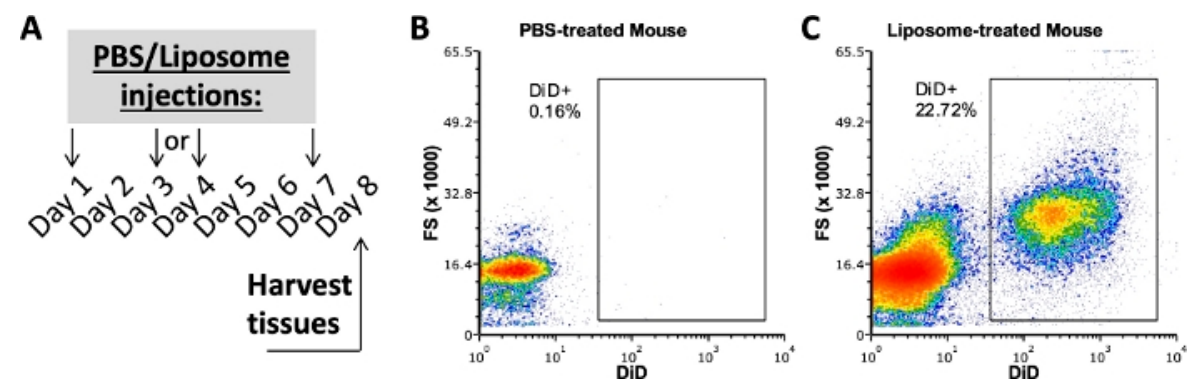

Figure 2: Representative DiD staining from PBS- or Liposome-treated mice.

(A) Experimental schematic for PBS and liposome treatments. PBS or liposomes were injected three times over the course of one week. Tissues were harvested on Day 8 of treatment. $(\mathbf{B}, \mathbf{C})$ Representative flow plots reveal positive DiD staining in liposome-treated (C), but not PBS-treated (B) mice. FSC, forward scatter. Please click here to view a larger version of this figure. 

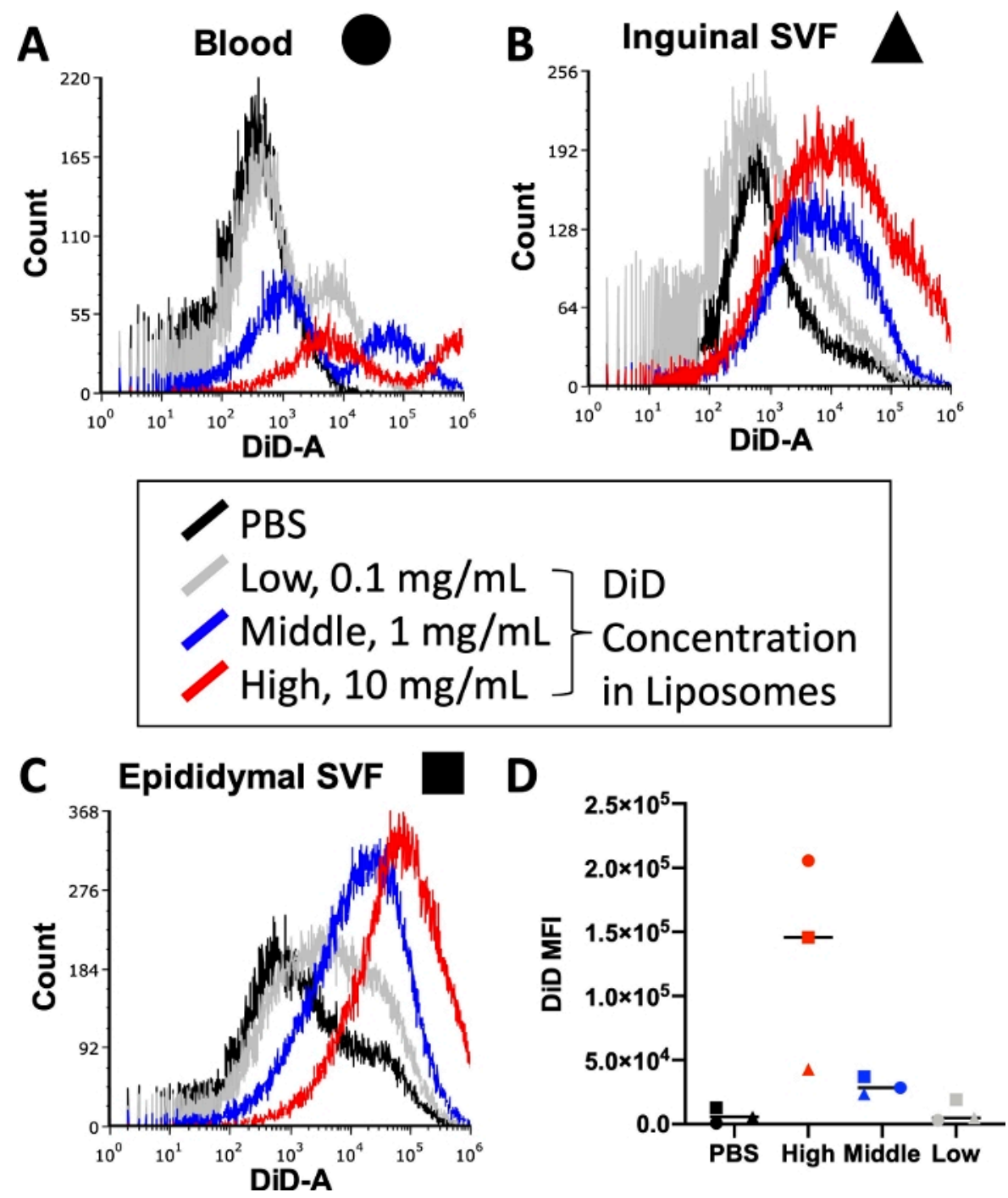

Figure 3: Titration of DiD in liposomes.

Liposomes were prepared with three different concentrations of DiD and injected into mice. Grey indicates the low concentration at $0.1 \mathrm{mg} \mathrm{DiD}$ per $1 \mathrm{~mL}$ of liposomes, blue indicates the middle concentration at $1 \mathrm{mg} \mathrm{DiD} / \mathrm{mL}$ liposomes, and red indicates the high concentration at $10 \mathrm{mg} \mathrm{DiD} / \mathrm{mL}$ liposomes. A PBS-treated mouse was used as a negative control (black). Blood (A, circle), inguinal adipose (B, triange), and epididymal adipose (C, square) were harvested $24 \mathrm{~h}$ postinjection and processed to isolate a single-cell suspension. These samples were run on a flow cytometer to the level of detectable DiD. Tissue-specific histograms with overlays of each treatment group are presented to demonstrate fluorescence intensity per concentration $(\mathrm{A}-\mathrm{C})$. The arithmetic mean of $\mathrm{DiD}$ was also quantified for each tissue and concentration and plotted (D). SSC = side scatter. Please click here to view a larger version of this figure. 


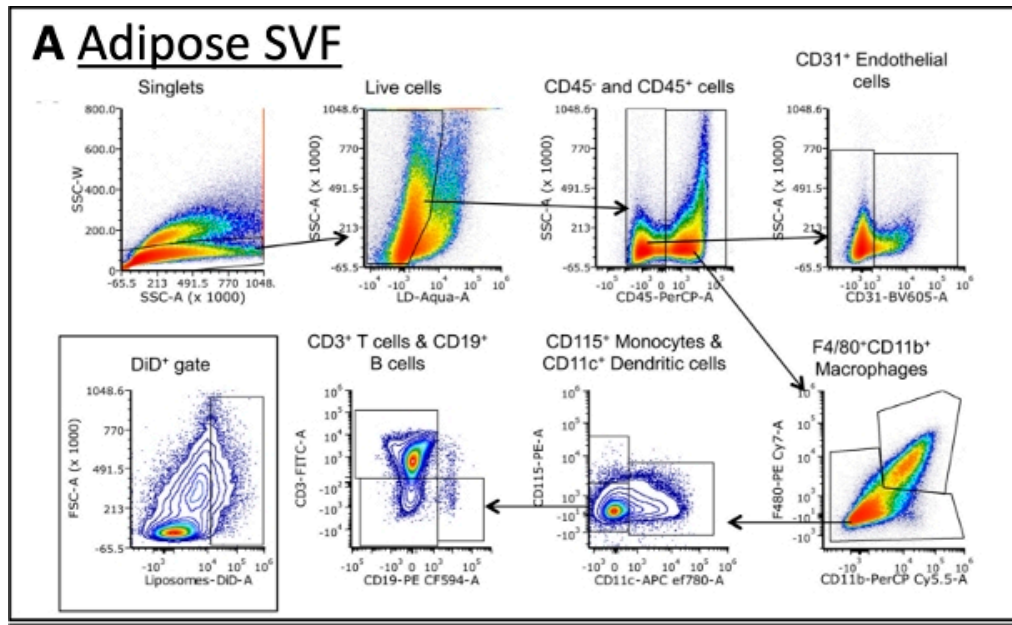

\section{B Spleen}
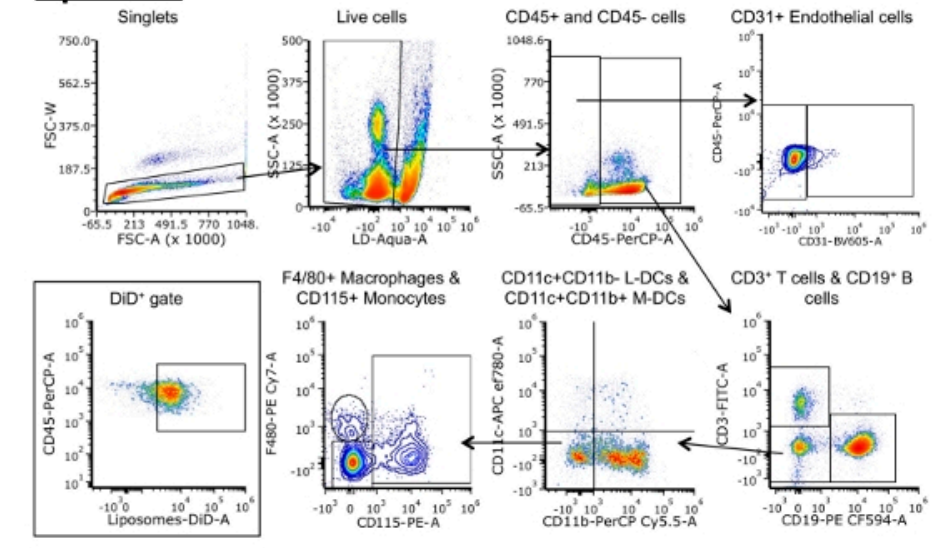

$\mathrm{F} 4 / 80+$ Macrophages \&
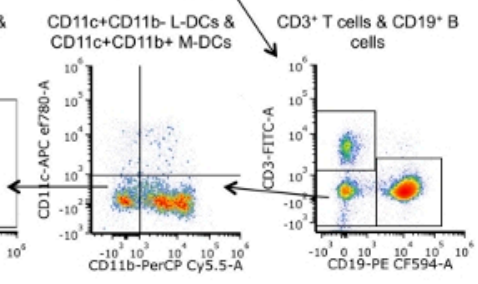

\section{Blood}

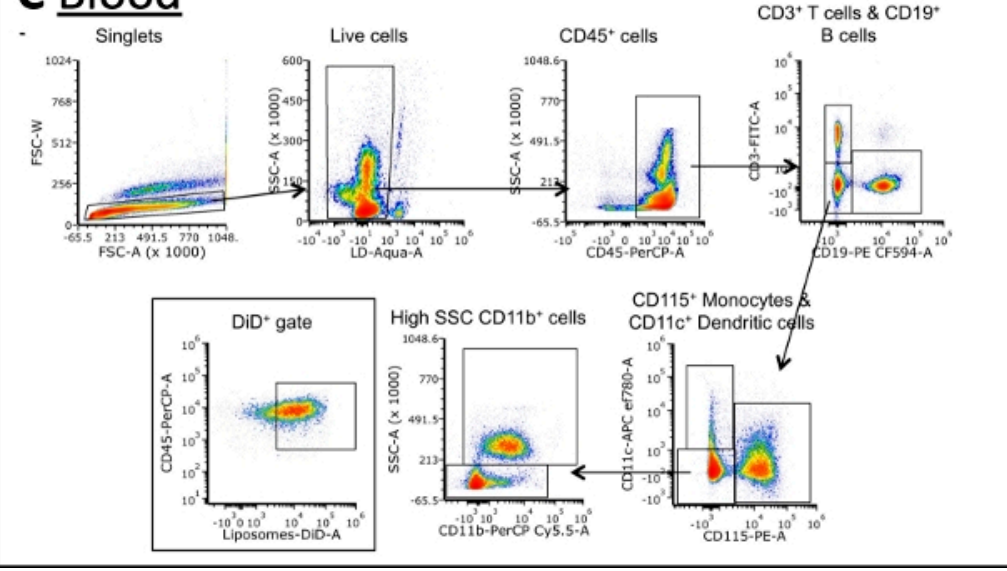

Figure 4: Representative flow cytometry analysis of cell subsets in adipose SVF, blood, and spleen.

(A-C) Schematic representative of gating strategy to identify cell subsets and $\mathrm{DiD}^{+}$cells in adipose SVF (A), spleen (B), and blood (C). Abbreviations: FSC = forward scatter; LD = live/dead; L-DCs = lymphoid dendritic cells; M-DCs = myeloid dendritic cells; SSC = side scatter. This figure is adapted from Osinski et al. ${ }^{3}$. Please click here to view a larger version of this figure. 


\begin{tabular}{|c|c|}
\hline Control & Purpose \\
\hline Mouse treated with PBS or saline & $\begin{array}{c}\text { Use the cells from this mouse for the } \\
\text { following flow cytometry controls: }\end{array}$ \\
\cline { 2 - 3 } & 1. Unstained cells \\
\cline { 2 - 3 } & 2. Live/dead single stain \\
\cline { 2 - 3 } & $\begin{array}{c}\text { 3. Cells stained with the full panel, but lacking } \\
\text { the liposome fluorescence to determine } \\
\text { positive liposome signal during analysis }\end{array}$ \\
\cline { 2 - 3 } & $\begin{array}{c}\text { This/these mouse/mice will also be used to determine } \\
\text { if liposomes have any effects in vivo as you will } \\
\text { have a non-liposome control in your experiment. }\end{array}$ \\
\hline Unloaded liposomes & $\begin{array}{c}\text { If you are loading a compound in your liposomes, } \\
\text { a portion of your liposome batch should be } \\
\text { synthesized without the compound. This accounts } \\
\text { for any in vivo effects of the liposomes alone. }\end{array}$ \\
\hline Fluorescence-minus-one (FMO) controls & $\begin{array}{c}\text { Since DiD can also be taken up by cellular membranes, } \\
\text { allocating some mice to receive free dye at an } \\
\text { amount equal to that found in the liposomes will help } \\
\text { account for any background membrane staining. }\end{array}$ \\
\hline \multirow{2}{*}{ DiD alone } & $\begin{array}{c}\text { These are cells stained with all but one of the antibodies in } \\
\text { true positive signal for that antibody during analysis }\end{array}$ \\
\hline
\end{tabular}

Table 1: Controls to use in this protocol. 


\begin{tabular}{|c|c|c|}
\hline Solution & Components & $\begin{array}{c}\text { Approximate volume } \\
\text { needed per batch/mouse }\end{array}$ \\
\hline \multicolumn{3}{|c|}{ Liposome preparation } \\
\hline Calcium acetate & $1 \mathrm{M}$ calcium acetate in $\mathrm{H}_{2} \mathrm{O}$ & $50 \mathrm{~mL}$ \\
\hline HEPES buffer & $10 \mathrm{mM}$ HEPES in $\mathrm{H}_{2} \mathrm{O}$, pH 7.4 & $50 \mathrm{~mL}$ \\
\hline Tesaglitazar in HEPES & in $10 \mathrm{mM}$ HEPES & $10 \mathrm{~mL}$ \\
\hline \multicolumn{3}{|c|}{ Tissue harvest, processing, and staining } \\
\hline Phosphate-buffered solution (PBS) & $\begin{array}{c}137 \mathrm{mM} \mathrm{NaCl}, 2.7 \mathrm{mM} \mathrm{KCl} \\
10 \mathrm{mM} \mathrm{Na}_{2} \mathrm{HPO}_{4}, 1.8 \mathrm{mM} \\
\mathrm{KH}_{2} \mathrm{PO}_{4} \text { in distilled } \mathrm{H}_{2} \mathrm{O}\end{array}$ & $2 \mathrm{~mL}$ \\
\hline PBS-Heparin & $0.1 \mathrm{mM}$ Heparin in PBS & $10 \mathrm{~mL}$ \\
\hline HEPES buffer & $20 \mathrm{mM}$ HEPES in PBS & $5 \mathrm{~mL}$ \\
\hline Digestion buffer & $\begin{array}{l}2 \mathrm{mg} / \mathrm{mL} \text { Collagenase } \\
\text { Type I in HEPES buffer }\end{array}$ & $5 \mathrm{~mL}$ \\
\hline AKC lysis buffer & $\begin{array}{c}0.158 \mathrm{M} \mathrm{NH}_{3} \mathrm{Cl}, 10 \mathrm{mM} \mathrm{KHCO}_{3}, 0.1 \\
\mathrm{mM} \mathrm{Na} 2 \mathrm{EDTA} \text { in } \mathrm{ddH}_{2} \mathrm{O}, \mathrm{pH} 7.2\end{array}$ & $15 \mathrm{~mL}$ \\
\hline FACS buffer & $1 \% \mathrm{BSA}, 0.05 \% \mathrm{NaN}_{3}$ in PBS & $15 \mathrm{~mL}$ \\
\hline Fc Block (diluted) & 1:50 Fc Block in FACS buffer & $250 \mu \mathrm{L}$ \\
\hline Fixation Buffer & $2 \%$ paraformaldehyde in PBS & $200 \mu \mathrm{L}$ \\
\hline
\end{tabular}

Table 2: Solutions to prepare. 


\begin{tabular}{|c|c|c|c|}
\hline A & B & C & D \\
\hline \multicolumn{4}{|c|}{ Extracellular Staining (2x antibody mix) } \\
\hline Antigen & Fluorophore & Ab volume per $100 \mu \mathrm{L}$ test & Total volume needed: \\
\hline CD45 & PerCP & $0.5 \mu \mathrm{L}$ & $\begin{array}{l}\text { Column C x } 1.2 \times \\
\text { Total \# samples }\end{array}$ \\
\hline CD11b & PerCP Сy5.5 & $0.25 \mu \mathrm{L}$ & $\begin{array}{c}(0.5 \mu \mathrm{L} / \text { test }) \times \\
(1.2) \times(\# \text { samples })\end{array}$ \\
\hline $\mathrm{F} 4 / 80$ & PE Cy7 & $0.25 \mu \mathrm{L}$ & $\begin{array}{c}(0.25 \mu \mathrm{L} / \text { test }) \times \\
(1.2) \times(\# \text { samples })\end{array}$ \\
\hline CD19 & PE-CF594 & $1 \mu \mathrm{L}$ & $\begin{array}{c}(0.25 \mu \mathrm{L} / \text { test }) \times \\
(1.2) \times(\# \text { samples })\end{array}$ \\
\hline CD3 & FITC & $1 \mu \mathrm{L}$ & $\begin{array}{c}(1.0 \mu \mathrm{L} / \text { test }) \times \\
(1.2) \times(\# \text { samples })\end{array}$ \\
\hline CD31 & BV605 & $0.25 \mu \mathrm{L}$ & etc... \\
\hline CD11c & APC ef780 & $1 \mu \mathrm{L}$ & \\
\hline CD115 & PE & $1.5 \mu \mathrm{L}$ & \\
\hline \multicolumn{4}{|c|}{$\begin{array}{l}\text { To create your antibody mix, combine the antibodies calculated in column D with FACS } \\
\text { buffer or Brilliant Violet Staining Buffer* to a final volume of ( } 50 \mu \mathrm{L} \times 1.2 \times \text { Total \# samples) }\end{array}$} \\
\hline \multicolumn{4}{|c|}{ Live/Dead staining (1x) } \\
\hline Live/Dead & Fluorophore & L/D volume per $200 \mathrm{uL}$ test & Total volume needed: \\
\hline Live/Dead & Aqua & $0.67 \mu \mathrm{L}$ & $\begin{array}{l}\text { Column C x } 1.2 \text { x } \\
\text { Total \# samples }\end{array}$ \\
\hline \multicolumn{4}{|c|}{ Intracellular Staining $(1 \mathrm{x})$} \\
\hline Antigen & Fluorophore & Ab volume per $50 \mu \mathrm{L}$ test & Total volume needed: \\
\hline aSMA & FITC & 0.125 & $\begin{array}{l}\text { Column C x } 1.2 \times \\
\text { Total \# samples }\end{array}$ \\
\hline
\end{tabular}


*Brilliant Violet Staining Buffer should be used if more than one antibody

conjugated to a Brilliant Violet fluorophore is being used in your panel.

Table 3: Example antibody panel and calculations of staining mixes to use for flow staining.

\section{Discussion}

Here we describe a three-part protocol to (i) prepare liposomes that are labeled with a fluorescent lipid dye and loaded with an anti-diabetic compound, tesaglitazar, (ii) administer liposomes to a mouse via retro-orbital injection, and (iii) harvest, process, and stain tissues to detect liposome uptake at a cellular level by flow cytometry. This protocol reviews preparation of approximately $150-\mu \mathrm{m}$ liposomes and assessment of uptake in adipose, blood, and the spleen. The liposome preparation is scalable, performed mostly at room temperature, and utilizes reverse-phase evaporation to maximize drug loading and removal of organic solvents. Using this protocol, up to $2 \mathrm{mg} / \mathrm{mL}$ tesaglitazar concentration can be achieved in the purified liposome sample. The prepared liposomes can be stored in HEPES buffer at 4 ${ }^{\circ} \mathrm{C}$ for over a year. In our experience, they demonstrated minimal variation of mean particle size. Under $10 \%$ of drug content loss was demonstrated spectrophotometrically, following ultrafiltration separation of liposomes from external drug with a $10 \mathrm{kDa}$ centrifugal filter.

During liposome preparation, there are some critical steps and factors to consider. First, the order of the protocol steps is important and must be adhered to. Second, the $\mathrm{pH}$ of the solution used when loading tesaglitazar must be maintained at 7.4 in order to maximize solubility and effective loading. Third, proper assembly of equipment and filters ensures that the output of each step is of the proper size and purity. For example, if $100-$ and $200-n m$ filters are not assembled properly, a more heterogenous and improperly- sized batch of liposomes may result. Fourth, complete removal of Ca-acetate prior to drug-loading is needed to maximize the transfer of tesaglitazar into the liposomes. To test for complete removal of Ca-acetate, use high-speed sedimentation to remove the liposomes and then measure Ca-acetate levels in the non-liposomal solution. Fifth, it is important to weigh and record the mass of all materials added to the liposome preparation at each step. This ensures that proper concentrations can be calculated and needed ratios of materials are maintained. Finally, if the technique is not properly executed, there may be an undesirable level of heterogeneity. It is important to thoroughly check this parameter using DLS and other approaches such as electron microscopy. To improve homogeneity, consider adjusting the selected filter size or stacking two filters.

Additionally, it is critical that controls and an antibody panel for flow cytometry are planned and optimized prior to conducting this protocol in full (Table 1, Table 3). Antibodies should be tested to ensure proper concentrations are used for staining and that overlap between fluorophores is minimal. The excitation and emission of the dye used during liposome preparation must also be factored into panel planning. In our results, we utilized DiD, which has a similar excitation and emission to fluorophores such as Allophycocyanin (APC) and AlexaFluor 647. Thus, we did not select antibodies conjugated to these fluorophores in our antibody panel. Furthermore, isotype controls are not included in this protocol. This is because the antibodies selected for this protocol are well-validated, commercially available antibodies. However, if 
interested in using an antibody that has not been optimized previously, please consider testing the antibody against an isotype control on the tissues of interest prior to conducting the full experiment.

While this protocol demonstrates how to extract and process the blood, spleen, inguinal adipose, and epididymal adipose tissues from the mouse post-treatment, this general approach can be applied to other tissues. Depending on the tissue of interest, processing and digestion protocols may need to be altered as is published for the following tissues: lung ${ }^{21}$, liver $^{22}$, peritoneal cavity ${ }^{3}$, bone marrow ${ }^{3}, 23$, brain $^{24}$.

An important limitation of this method to consider is that uptake can only be assessed at one time point per animal. Thus, it may be advantageous to couple this protocol with other non-invasive imaging techniques or plan accordingly to ensure sufficient resources for conducting the assessment. Timing of cellular uptake and cellular turn over are important factors to consider: liposomes will circulate throughout the body in the first $24 \mathrm{~h}$ and depending on the lifespan of the cells that take up liposomes or how they respond to uptake, cell death or further phagocytosis may occur. Our previous study demonstrated changes in the population characteristics of $\mathrm{DiD}^{+}$populations at different time points ${ }^{3}$. For that reason, evaluating uptake at earlier time points or time points most relevant to the biology of mechanism of interest is important. Additionally, while quantification of cell uptake in the entire tissue can be performed with this protocol, flow cytometry cannot reveal tissue localization. Coupling this approach with histological methods can help to address this limitation.

In general, this protocol complements existing methodology such as histology and whole-body fluorescence imaging. With the continued advancements in flow cytometry tools and methods, the development of larger panels to more and more specific cell populations will become possible. We suggest that this protocol be used in addition to the aforementioned methods as this will improve the evaluation of cellular uptake and also provide the opportunity to validate the outcomes observed by flow cytometry. For example, should it be found that a majority of the particles in adipose tissue were taken up by macrophages by flow cytometry. Immunofluorescence of an additional aliquot of the same adipose tissue could be saved, fixed, sectioned, and stained for macrophage markers to verify that the cell type does indeed take up liposomes. This approach should add rigor to nanoparticle biodistribution assays conducted: validating cellspecific targeting, quantifying cellular uptake, identifying offtarget uptake, and hopefully providing information to generate mechanistic hypotheses for observed therapeutic outcomes. This protocol can be also be adapted for future studies using different liposomes, investigating uptake in other tissues, and testing new compounds in the setting of obesity and dysmetabolism or any other disease in which nanoparticledelivery is a feasible therapeutic option.

\section{Disclosures}

The authors have nothing to disclose.

\section{Acknowledgments}

The authors would like to acknowledge Michael Solga and the rest of the Flow Cytometry Core staff for providing flow cytometry training and services. The authors would also like to acknowledge Shiva Sai Krishna Dasa, Dustin K. Bauknight, Melissa A. Marshall, James C. Garmey, Chantel McSkimming, Aditi Upadhye, and Prasad Srikakulapu for their assistance with liposome preparation (SSKD, DKB), tissue harvests (MAM, JCG), and flow cytometry staining and sample acquisition (AU, PS, CM). This work was supported 
by AstraZeneca, R01HL 136098, R01HL 141123 and R01HL 148109, AHA 16PRE30770007, and T32 HL007284 grants.

\section{References}

1. Allen, T.M., Cullis, P.R. Liposomal drug delivery systems: from concept to clinical applications. Advanced Drug Delivery Reviews. 65 (1), 36-48 (2013).

2. Sercombe, L. et al. Advances and Challenges of Liposome Assisted Drug Delivery. Frontiers in Pharmacology. 6, 286 (2015).

3. Osinski, V. et al. In vivo liposomal delivery of PPARa/Y dual agonist tesaglitazar in a model of obesity enriches macrophage targeting and limits liver and kidney drug effects. Theranostics. 10 (2) (2020).

4. Bauknight, D.K. et al. Importance of thorough tissue and cellular level characterization of targeted drugs in the evaluation of pharmacodynamic effects. PLOS ONE. 14 (11) (2019).

5. Rosales, C., Uribe-Querol, E. Phagocytosis: A Fundamental Process in Immunity. BioMed Research International. (2017).

6. He, H., Ghosh, S., Yang, H. Nanomedicines for dysfunctional macrophage-associated diseases. Journal of Controlled Release. 247, 106-126 (2017).

7. Song, G., Petschauer, J.S., Madden, A.J., Zamboni, W.C. Nanoparticles and the mononuclear phagocyte system: pharmacokinetics and applications for inflammatory diseases. Current Rheumatology Reviews. 10 (1), 22-34, at <https://www.ncbi.nlm.nih.gov/ pubmed/25229496> (2014).

8. Litzinger, D.C., Buiting, A.M.J., van Rooijen, N., Huang, L. Effect of liposome size on the circulation time and intraorgan distribution of amphipathic poly(ethylene glycol)-containing liposomes. BBA - Biomembranes. (1994)

9. Su, C., Liu, Y., He, Y., Gu, J. Analytical methods for investigating in vivo fate of nanoliposomes: A review. Journal of Pharmaceutical Analysis. (2018).

10. Vasquez, K.O., Casavant, C., Peterson, J.D. Quantitative whole body biodistribution of fluorescent-labeled agents by non-invasive tomographic imaging. PLoS One. 6 (6), e20594 (2011).

11. Larmann, J. et al. In vivo fluorescence-mediated tomography for quantification of urokinase receptordependent leukocyte trafficking in inflammation. Anesthesiology. 113 (3), 610-618 (2010).

12. Feng, B. et al. Clodronate liposomes improve metabolic profile and reduce visceral adipose macrophage content in diet-induced obese mice. PLoS One. 6 (9), 1-11 (2011).

13. Bu, L., Gao, M., Qu, S., Liu, D. Intraperitoneal injection of clodronate liposomes eliminates visceral adipose macrophages and blocks high-fat diet-induced weight gain and development of insulin resistance. The AAPS Journal. 15 (4), 1001-1011 (2013).

14. Toita, R., Kawano, T., Murata, M., Kang, J.H. Antiobesity and anti-inflammatory effects of macrophagetargeted interleukin-10-conjugated liposomes in obese mice. Biomaterials. 110, 81-88 (2016).

15. Sakurai, Y., Kajimoto, K., Hatakeyama, H., Harashima, H. Advances in an active and passive targeting to tumor and adipose tissues. Expert Opin Drug Deliv. 12 (1), 41-52 (2015). 
16. Nakhlband, A. et al. Combating atherosclerosis with targeted nanomedicines: Recent advances and future prospective. Biolmpacts. (2018).

17. Sibuyi, N.R.S., Meyer, M., Onani, M.O., Skepu, A., Madiehe, A.M. Vascular targeted nanotherapeutic approach for obesity treatment. International Journal of Nanomedicine. (2018).

18. Honig, M.G., Hume, R.I. Fluorescent carbocyanine dyes allow living neurons of identified origin to be studied in long-term cultures. Journal of Cell Biology. (1986).

19. Warren, J.S.A., Feustel, P.J., Lamar, J.M. Combined Use of Tail Vein Metastasis Assays and Real-Time In Vivo Imaging to Quantify Breast Cancer Metastatic Colonization and Burden in the Lungs. Journal of visualized experiments : JoVE. (2019).

20. Dasa, S.S.K. et al. Plectin-targeted liposomes enhance the therapeutic efficacy of a PARP inhibitor in the treatment of ovarian cancer. Theranostics. (2018).

21. Morrison, B.E., Park, S.J., Mooney, J.M., Mehrad, B. Chemokine-mediated recruitment of NK cells is a critical host defense mechanism in invasive aspergillosis. Journal of Clinical Investigation. (2003).

22. Finlon, J.M., Burchill, M.A., Jirón Tamburini, B.A. Digestion of the murine liver for a flow cytometric analysis of lymphatic endothelial cells. Journal of Visualized Experiments. (2019).

23. Upadhye, A. et al. Diversification and CXCR4-Dependent Establishment of the Bone Marrow B-1a Cell Pool Governs Atheroprotective IgM Production Linked to Human Coronary Atherosclerosis. Circulation research. (2019).
24. O'Brien, C.A., Harris, T.H. ICOS-deficient and ICOS YF mutant mice fail to control Toxoplasma gondii infection of the brain. PLOS ONE. (2020). 